\title{
Comparison of five RNA extraction methods from rabbit's blood
}

\author{
Afif M. Abdel Nour ${ }^{*}$, Elie K. Barbour ${ }^{2}$, Flore Depint ${ }^{1}$, Maxime Dooms ${ }^{1}$, Khady Niang ${ }^{1}$, \\ Amélie Dulac ${ }^{1}$, Claude-Narcisse Niamba ${ }^{1}$, Ghenwa Chaaya ${ }^{1}$, and Philippe R. Pouillart ${ }^{1}$ \\ ${ }^{1}$ Institut Polytechnique LaSalle Beauvais, Beauvais, France \\ ${ }^{2}$ Department of Animal and Veterinary Science, Faculty of Agriculture and Food Sciences \\ (FAFS), American University of Beirut (AUB), Beirut, Lebanon
}

\begin{abstract}
Extraction of RNA from eukaryotic cells is a crucial stage in many molecular biology studies. Thus it is essential to use the most appropriate method that maximizes the yield and maintains the integrity of extracted RNA. The present work compared five manual RNA extraction methods of different dilutions of rabbit blood. The assessment of the concentration and purity of the extracted RNA were used as parameters to evaluate the efficiency of each method. Statistical analysis showed that the most effective method for RNA extraction from rabbit blood is by following the protocol of the RNeasy Lipid Tissue kit, but it requires the use of toxic reagents. The most efficient kit using no toxic reagents is the RNeasy protect animal blood.
\end{abstract}

Keywords: Non-toxic method, chloroform, phenol, blood, efficiency, rabbit, RNA extraction.

\section{INTRODUCTION}

The success of any RNA-based analysis (e.g. reverse transcription $\mathrm{PCR}$, real time $\mathrm{PCR}$, microarrays, in vitro translation analysis) reflects the accuracy of gene expression evaluation and depends to a great extent on the yield, purity and the integrity of the extracted RNA (Bustin, Benes, Nolan, and Pfaffl, 2005; Imbeaud and Auffray, 2005; Raeymaekers, 1993). The operator should have complete control on the steps during the handling and processing of the sample, since RNA recovery amounts and the sensitivity of extracted material could be jeopardised by the following protocols (Perez-Novo, Claeys, Speleman, Van Cauwenberge, Bachert, and Vandesompele, 2005).

In the last few years, RNA analysis has been an important part in an increasingly large number of research studies. Different extraction methods can yield different RNA quantities and qualities. Changing the type of homogenization alone can also yield different RNA quality (Fleige and Pfaffl, 2006). The origin of RNA (e.g. from human, animal or plant), the sampling procedures (biopsy material, single cell sampling, laser micro-dissection, tissue) as well as the method of RNA isolation (total RNA or polyadenylated RNA methods) differ very often from one laboratory to another (Bustin et al., 2005; Pfaffl, 2004). As a matter of example the use of liquid extraction by Trizol gives different results (low quality) when compared to column based extraction method (Santiago-Vazquez, Ranzer, and Kerr, 2006).
Furthermore, the use of kits containing phenol (such as Trizol ${ }^{\circledR}$ or Qiazol ${ }^{\circledR}$ ) is risky. To use such kits, it is recommended to use a fume cupboard with specific filters (organic solvents catcher). Furthermore, these methods also involve the use of chloroform, toxin acting on central nervous system, being transformed into phosgene, a more powerful toxic. Even under $1500 \mathrm{ppm}$, chloroform can induce headache, weariness. Considering a chronically use, some cases of liver and kidney diseases are reported (Pichard, Bisson, Diderich, Dujardin, Lacroix, Lamy, Lefevre, Magaud, Pepin, and Villey, 2005). Phenol is reported as a toxic but currently non-carcinogenic. Chloroform is both toxic and carcinogenic.

It is common for RNA extraction methods to be modified and optimised for specific clinical specimen or downstream use (Akin, Wu, and Lin, 1998; Hale, Green, and Brown, 1996; Santti, Hyypia, and Halonen, 1997). Most commercial RNA extraction methods are optimised for human blood samples. Many differences exist in the blood composition of the species belonging to Animalia Kingdom.

The aim of this work is to compare five RNA extraction methods and to determine the optimal efficient method to extract RNA from rabbits' whole blood, and compare methods involving chloroform/phenol with other ones. In assessing the quality and the quantity of the extracted RNA, the spectrophotometer method with Helma UV cell will be used (Eppendorf, Paris, France). 


\section{MATERIAL AND METHODS}

Whole blood was collected from the jugular vein of 65-days-old immunocompetent healthy rabbits (Oryctolagus cuniculus, Hycole, Marcoing, France), in heparinized tubes (Sarstedt, Germany), except for the RNeasy Protect animal kit, where the blood must be used without any additive. Aliquots were diluted in saline water at 10, 100 and 1000-fold. The three dilutions, in addition to undiluted blood samples were included in the RNA extraction procedures.

Five different manual methods were used for RNA extraction from $500 \mu \mathrm{l}$ aliquots of each of the three sample dilutions and undiluted blood (Table 1). The following commercially available kits were compared: TRIzol - LS Reagent (\# 10296-010, Invitrogen, Cergy Pontoise, France), QIAamp RNA Blood Mini Kit (\# 52304, Qiagen ,Courtabeouf, France); RiboPureBlood Kit (\# AM1928, Applied Biosystems, Les Uliss, France) and RNeasy Protect Animal Blood Kit (\# 73224, Qiagen, Courtaboeuf, France). All extractions were performed according to the user's manual provided by the manufacturers.

For each dilution, three extractions were performed on three independent days (D) by two independent operators (O) according to a defined schedule (D1O1, D2O1, D3O2) and in triplicate, excepted for the RNeasy Protect animal blood kit, because the dilutions must be performed before the blood coagulates. The extracts were then assessed by spectrophotometry. Optical density (OD) at 260 and $280 \mathrm{~nm}$ were measured in triplicate to estimate RNA concentration and quality. The differences between the means were assessed by two-tailed t-test. The assumptions of normal distributions with equality of variances were verified before the comparison of means using Shapiro-Wilk normality test and a Test of Levene. An attained significance level or P-value below 0.05 was considered significant. Statview version 5.0 (SAS institute, Grégy-sur-Yerres, France) was used for the statistical analyses.

Table 1: Comparison of the DNA yield and quality following the different extraction protocols.

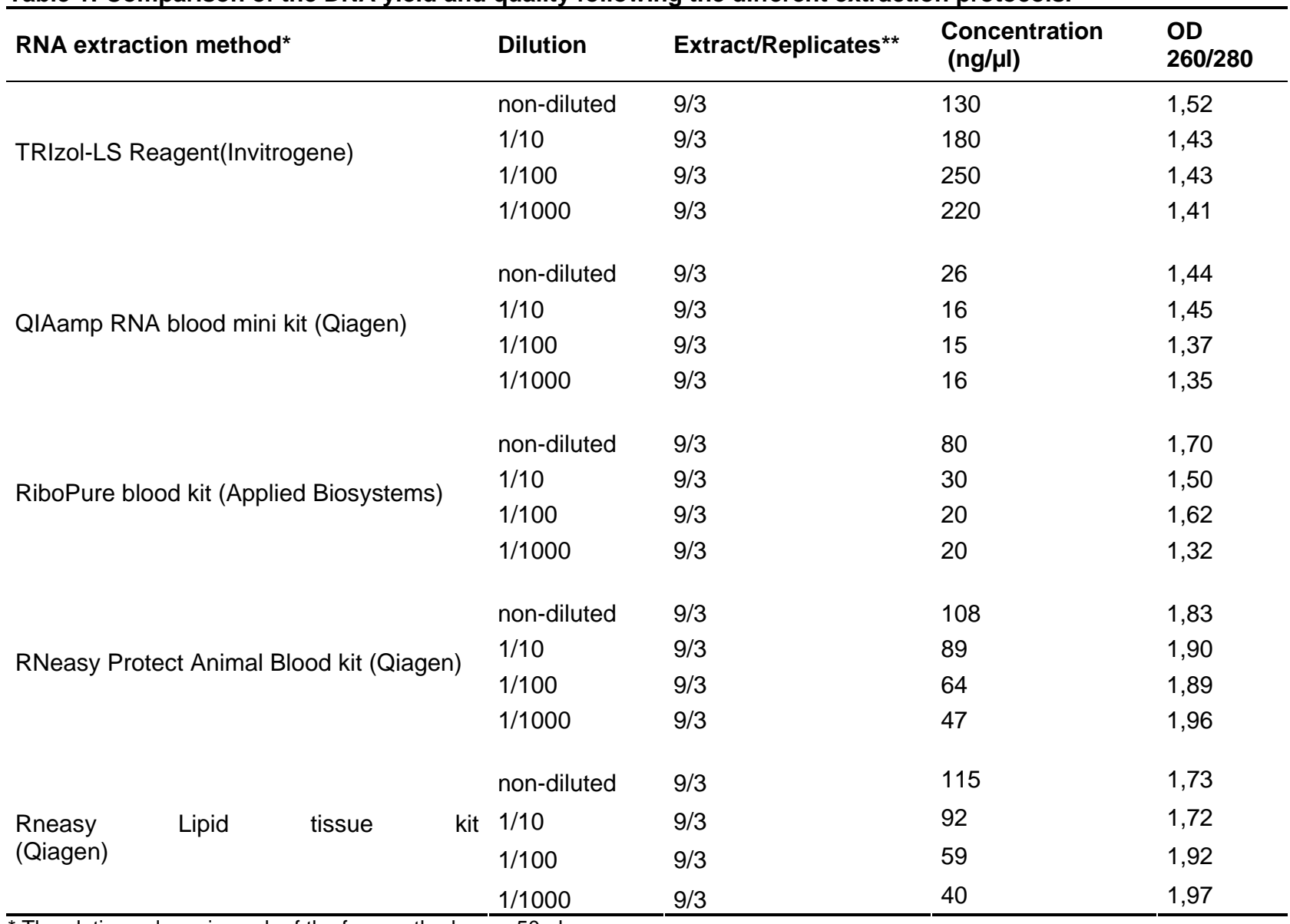

* The elution volume in each of the four methods was $50 \mu \mathrm{l}$.

** Each extracts was run in triplicate leading to 27 observations per dilution per method of extraction. 


\section{RESULTS AND DISCUSSION}

The differences between the means were evaluated by 27 measurements per dilution of the original blood sample for the four first kits and by 2 measurements per dilution for the last one. The means optical densities (at $260 \mathrm{~nm}$ ) for the non-diluted samples were $1.523 \pm 0.015,1.440 \pm 0.023,1.703 \pm 0.075$, $1.733 \pm 0.040$ and $1,79 \pm 0.015$ respectively for the TRIzol - LS Reagent, QIAamp RNA Blood Mini Kit, RiboPure-Blood Kit, the Rneasy Lipid tissue kit and RNeasy Protect Animal Blood kit. Hence, Table 1 showed a better RNA quality from non-diluted blood extracted with the RNeasy Protect Animal Blood kit, Ribopure or RNeasy lipid tissue kit (OD260/280 = $1.83 ; 1.70$ and 1.73 , respectively) when compared to the other extractions methods $(O D 260 / 280=1.44$, 1.52 and 1,60). No significant differences were observed between the Ribopure and the RNeasy lipid tissue kit extraction for the undiluted sample extracts $(P=0.295)$. For all kits except RNeasy Lipid Tissue kit and RNeasy Protect Animal Blood kit, the optimal purity is with the undiluted samples. The three first extraction techniques thus lead to relatively poor quality RNA (OD ratio below 1.8 indicates the presence of contaminants in the sample). The very high RNA concentration measured by TRizol extraction is most likely due to protein contaminants. The RNeasy Protect Animal Blood kit gave us the best results regarding quantity, quality and safety.

These relatively moderate results for these extraction methods can be explained by differences between blood compositions of the two species (human and rabbit). To our knowledge this is the first study comparing different kits for the RNA extraction from rabbit's blood. Although these kits were optimised for extracting RNA from human blood, some of these kits showed relatively good results. Trizol yielded high RNA levels but a moderate purity, which can be optimised by purifying the RNA on silica column.

Table 1 shows that the RNeasy Protect animal blood kit can be considered the optimum extraction method in this setting, qualitatively, and quantitatively.

Moreover, this method can be semi-automated with the QIAcube, thus avoiding manipulation errors (and increasing repeatability) and is less time consuming, allowing the extraction of 12 samples in 35 min cycle. An additional benefit to the extraction using QIAcube is the fact that the whole process is integrated, limiting the transfer of samples and the use of other equipments (e.g. centrifuge, hoods, etc.) that are required for the manual extractions.
Conflict of interest statement: None of the authors of this paper has a financial or personal relationship with other people or organizations that could inappropriately influence or bias the content of the paper.

\section{REFERENCES}

Akin, A., Wu, C. C., \& Lin, T. L. (1998). A comparison of two RNA isolation methods for double-stranded RNA of infectious bursal disease virus. J Virol Methods, 74, 179-184.

Bustin, S. A., Benes, V., Nolan, T., \& Pfaffl, M. W. (2005). Quantitative real-time RT-PCR--a perspective. J Mol Endocrinol, 34, 597-601.

Fleige, S., \& Pfaffl, M. W. (2006). RNA integrity and the effect on the real-time qRT-PCR performance. Mol Aspects Med, 27, 126-139.

Hale, A. D., Green, J., \& Brown, D. W. (1996). Comparison of four RNA extraction methods for the detection of small round structured viruses in faecal specimens. $J$ Virol Methods, 57, 195-201.

Imbeaud, S., \& Auffray, C. (2005). 'The 39 steps' in gene expression profiling: critical issues and proposed best practices for microarray experiments. Drug Discov Today, 10, 1175-1182.

Perez-Novo, C. A., Claeys, C., Speleman, F., Van Cauwenberge, P., Bachert, C., \& Vandesompele, J. (2005). Impact of RNA quality on reference gene expression stability. Biotechniques, 39, 54-56.

Pfaffl, M. W. (2004). Quantification Strategies in real-time PCR. In S. A. Bustin (Ed.), The Real-Time PCR Encyclopedia A-Z of Quantitative PCR (pp. 89-113). La Jolla, CA: International University line.

Pichard, A., Bisson, M., Diderich, R., Dujardin, R., Lacroix, G., Lamy, M. H., Lefevre, J. P., Magaud, H., Pepin, G., \& Villey, C. (2005). Chlorophorme. In INERIS (Ed.), Fiche de données toxicologiques et environnementales des substances chimiques (pp. 149): L'Institut National de l'environnement industriel et des risques.

Raeymaekers, L. (1993). Quantitative PCR: theoretical considerations with practical implications. Anal Biochem, 214, 582-585.

Santiago-Vazquez, L. Z., Ranzer, L. K., \& Kerr, R. G. (2006). Comparison of two total RNA extraction protocols using the marine gorgonian coral Pseudopterogorgia elisabethae and its symbiont Symbiodinium sp. Electron J Biotechnol, 9, 598-603.

Santti, J., Hyypia, T., \& Halonen, P. (1997). Comparison of PCR primer pairs in the detection of human rhinoviruses in nasopharyngeal aspirates. $\mathrm{J}$ Virol Methods, 66, 139-147. 